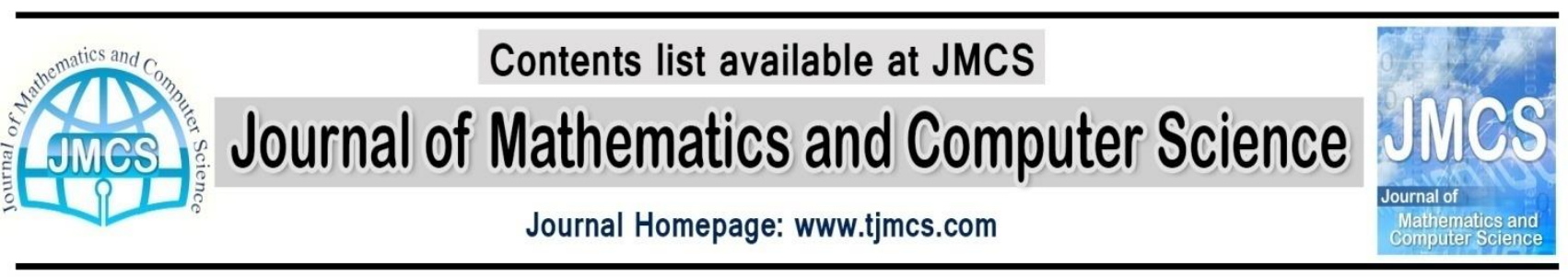

\title{
Fixed Point Results in Partial Metric Spaces Using Generalized Weak Contractive Conditions
}

\author{
M. Akram*, W. Shamaila ${ }^{\dagger}$ \\ *Department of Mathematics GC University, Lahore, Pakistan. \\ dr.makram@gcu.edu.pk \\ ${ }^{\dagger}$ Department of Mathematics, Kinnaird College for Women, Lahore, Pakistan. \\ shamailawaheed20@gmail.com
}

Article history:

Received July 2014

Accepted September 2014

Available online October 2014

\section{Abstract}

In this work, fixed point results using generalized weakly contractive conditions on partial metric spaces are presented. These results generalize many previously obtained results. Some examples are also given to show the usability of these results.

Keywords: Partial Metric, Generalized Weak contractive condition, Fixed point.

\section{Introduction}

The concept of partial metric spaces was given by Steve Matthews [1, 2] in 1992 to study the denotational semantics of data flow network. He presented partial metric space as a generalization of metric space in the sense that the self distance of any point need not be zero. Recently many authors have focused on the fixed point results in partial metric spaces (see e.g.[3-9]).

The notion of $\phi$-contraction was introduced by Boyd and Wong [10] and the weak $\phi$-contraction was introduced by Alber and Guerre-Delabriere [11] as a generalization of $\phi$-contraction. Later on $\phi$ contractions and weak $\phi$-contractions have been studied by many authors (see e.g. $[5-9,12,14]$ ) in metric spaces as well as in partial metric spaces.

Consistent with Matthews [1, 2], Karapinar [3] and Altun and Erduron [4] some important definitions and results which are used in this paper are given in the following. 
Definition 1.1 [1,2] A partial metric " $p$ " on $X$ is a function from $X \times X$ to $R^{+}$such that for every element $x, y$ and $z$ of $X$ it satisfies following axioms.

$$
\begin{aligned}
& p_{1}: 0 \leq p(x, x) \leq p(x, y) . \\
& p_{2}: p(x, x)=p(x, y)=p(y, y) \text { if and only if } x=y . \\
& p_{3}: p(x, y)=p(y, x) . \text { (symmetry) } \\
& p_{4}: p(x, z) \leq p(x, y)+p(y, z)-p(y, y) . \text { (triangular inequality) }
\end{aligned}
$$

If " $p$ " is a partial metric on $X$ then $(X, p)$ is called a partial metric space (PMS).

For a partial metric $p$ on $X$, the function $d_{p}: X \times X \rightarrow R^{+}$defined by $d_{p}(x, y)=2 p(x, y)-p(x, x)-p(y, y)$ for all $x, y, z \in X$ is a metric on $X$. Each partial metric " $p$ " on $X$ generates a $T_{0}$ topology $\tau_{p}$ on $X$ for which the collection $\left\{B_{p}(x, \varepsilon): x \in X, \varepsilon>0\right\}$ of all open balls forms a base. Where $B_{p}(x, \varepsilon)=\{y \in X: p(x, y)<p(x, x)+\varepsilon\}$ for each $\varepsilon>0$ and $x \in X$.

Definition 1.2 [1, 2, 4]

1. A sequence $\left\{y_{n}\right\}$ in a partial metric space $(X, p)$ converges to the limit $y \in X$ if and only if $\lim _{n \rightarrow \infty} p\left(y, y_{n}\right)=p(y, y)$.

2. A sequence $\left\{y_{n}\right\}$ in a partial metric space $(X, p)$ is called Cauchy if and only if $\lim _{m, n \rightarrow \infty} p\left(y_{m}, y_{n}\right)$ exists and is finite.

3. A partial metric space $(X, p)$ is said to be complete if every Cauchy sequence $\left\{y_{n}\right\}$ in $X$ converges, with respect to $\tau_{p}$, to a point $y \in X$ such that $\lim _{m, n \rightarrow \infty} p\left(y_{m}, y_{n}\right)=p(y, y)$.

4. The mapping $f: X \rightarrow X$ is said to be continuous at $y_{0} \in X$, if for every $\varepsilon>0$, there exists $\delta>0$ such that $f\left(B_{p}\left(y_{0}, \delta\right)\right) \subset B_{p}\left(f\left(y_{0}\right), \varepsilon\right)$.

The following lemma will be frequently used in the proofs of the main results.

Lemma $1.3[1,4]$ A sequence $\left\{y_{n}\right\}$ is a Cauchy sequence in a partial metric space $(X, p)$ if and only if it is a Cauchy sequence in the metric space $\left(X, d_{p}\right)$.

I. A partial metric space $(X, p)$ is complete if and only if the metric space $\left(X, d_{p}\right)$ is complete. Moreover, $\lim _{n \rightarrow \infty} d_{p}\left(y, y_{n}\right)=0$, if and only if $p(x, x)=\lim _{n \rightarrow \infty} p\left(y, y_{n}\right)=\lim _{n, m \rightarrow \infty} p\left(y_{n}, y_{m}\right)$. Where $y$ is the limit of $\left\{y_{n}\right\}$ in $\left(X, d_{p}\right)$. 
II. Let $X$ be a complete partial metric space. Then

(a) If $p(x, y)=0$, then $x=y$.

(b) If $x \neq y$, then $p(x, y)>0$.

III. Let $X$ be a partial metric space. Assume that the sequence $\left\{y_{n}\right\}$ is converging to $z$ as $n \rightarrow \infty$. such that $p(z, z)=0$. Then $\lim _{n \rightarrow \infty} p\left(y_{n}, y\right)=p(z, y)$ for all elements $y$ of $X$.

\section{Main results}

In the following theorem a generalized form of weak $\phi$-contraction is used.

Theorem 2.1 Let $(X, p)$ be a complete partial metric space and $T: X \rightarrow X$ be a self map such that for all $x, y \in X$

$$
p(T x, T y) \leq M(x, y)-\phi(M(x, y))
$$

where

$$
M(x, y)=\max \left\{p(x, y), \frac{1}{2}[p(x, T x)+p(y, T y)], \frac{1}{2}[p(x, T y)+p(y, T x)]\right\}
$$

and $\phi:[0, \infty) \rightarrow[0, \infty)$ is continuous non-decreasing function with $\phi(t)=0$ if and only if $t=0$. Then $T$ has a unique fixed point.

Proof: Let $y_{0} \in X$ be fixed. Define a sequence $\left\{y_{n}\right\}$ in $X$ by $y_{n+1}=T y_{n}$, for all $n \geq 0$. If there exist a positive integer $n_{0}$ such that $p\left(y_{n_{0}+1}, y_{n_{0}}\right)=0$ or $p\left(T y_{n_{0}}, y_{n_{0}}\right)=0$, then $T y_{n_{0}}=y_{n_{0}}$, this shows that $y_{n_{0}}$ is the fixed point of $T$. Hence we assume that $p\left(T y_{n}, y_{n}\right)=p\left(y_{n+1}, y_{n}\right) \neq 0$, for all $n \geq 0$. By substituting $x=y_{n}$ and $y=y_{n+1}$ in (1), we have

$$
p\left(T y_{n}, T y_{n+1}\right)=p\left(y_{n+1}, y_{n+2}\right) \leq M\left(y_{n}, y_{n+1}\right)-\phi\left(M\left(y_{n}, y_{n+1}\right)\right)
$$

where

$$
M\left(y_{n}, y_{n+1}\right)=\max \left\{\begin{array}{l}
p\left(y_{n}, y_{n+1}\right), \frac{1}{2}\left[p\left(y_{n}, y_{n+1}\right)+p\left(y_{n+1}, y_{n+2}\right)\right], \\
\frac{1}{2}\left[p\left(y_{n}, y_{n+2}\right)+p\left(y_{n+1}, y_{n+1}\right)\right]
\end{array}\right\} .
$$

By $p_{4}, p\left(y_{n}, y_{n+2}\right)+p\left(y_{n+1}, y_{n+1}\right) \leq p\left(y_{n}, y_{n+1}\right)+p\left(y_{n+1}, y_{n+2}\right)$

If $p\left(y_{n}, y_{n+1}\right)<\frac{1}{2}\left[p\left(y_{n}, y_{n+1}\right)+p\left(y_{n+1}, y_{n+2}\right)\right]$, then 


$$
M\left(y_{n}, y_{n+1}\right)=\frac{1}{2}\left\{p\left(y_{n}, y_{n+1}\right)+p\left(y_{n+1}, y_{n+2}\right)\right\}
$$

From (2) we have

$$
\begin{aligned}
p\left(y_{n+1}, y_{n+2}\right) \leq \frac{1}{2}\left[p\left(y_{n}, y_{n+1}\right)\right. & \left.+p\left(y_{n+1}, y_{n+2}\right)\right]-\phi\left(\frac{1}{2}\left[p\left(y_{n}, y_{n+1}\right)+p\left(y_{n+1}, y_{n+2}\right)\right]\right) \\
& <\frac{1}{2}\left[p\left(y_{n}, y_{n+1}\right)+p\left(y_{n+1}, y_{n+2}\right)\right]
\end{aligned}
$$

Which implies

$$
p\left(y_{n+1}, y_{n+2}\right) \leq p\left(y_{n}, y_{n+1}\right)
$$

If

$$
\frac{1}{2}\left[p\left(y_{n}, y_{n+1}\right)+p\left(y_{n+1}, y_{n+2}\right)\right]<p\left(y_{n}, y_{n+1}\right),
$$

then $M\left(y_{n}, y_{n+1}\right)=p\left(y_{n}, y_{n+1}\right)$ and again from (2), we have

$$
\begin{aligned}
p\left(y_{n+1}, y_{n+2}\right) \leq & p\left(y_{n}, y_{n+1}\right)-\phi\left(p\left(y_{n}, y_{n+1}\right)\right) \\
& <p\left(y_{n}, y_{n+1}\right)
\end{aligned}
$$

Hence

$$
p\left(y_{n+1}, y_{n+2}\right) \leq p\left(y_{n}, y_{n+1}\right)
$$

Thus in both cases we have $p\left(y_{n+1}, y_{n+2}\right) \leq p\left(y_{n}, y_{n+1}\right)$ for all $n$. Hence $\left\{p\left(y_{n}, y_{n+1}\right)\right\}$ is monotone decreasing sequence of non-negative real numbers so there exists a real number $r \geq 0$, such that

$$
\lim _{n \rightarrow \infty} p\left(y_{n}, y_{n+1}\right)=r
$$

Letting $n \rightarrow \infty$, in (3) or in (5), using (7) and regarding the continuity of $\phi$ we have $r \leq r-\phi(r)$, which forces $r=0$. Hence, in both cases

$$
\lim _{n \rightarrow \infty} p\left(y_{n}, y_{n+1}\right)=0
$$

Now consider, 


$$
\begin{aligned}
p\left(T y_{m-1}, T y_{m-1}\right)= & p\left(y_{m}, y_{m}\right) \leq M\left(y_{m-1}, y_{m-1}\right)-\phi\left(M\left(y_{m-1}, y_{m-1}\right)\right) \\
= & \max \left\{p\left(y_{m-1}, y_{m-1}\right), \frac{1}{2}\left[p\left(y_{m-1}, y_{m}\right)+p\left(y_{m-1}, y_{m}\right)\right]\right\} \\
& -\phi\left(\max \left\{p\left(y_{m-1}, y_{m-1}\right), \frac{1}{2}\left[p\left(y_{m-1}, y_{m}\right)+p\left(y_{m-1}, y_{m}\right)\right]\right\}\right) \\
= & \max \left\{p\left(y_{m-1}, y_{m-1}\right), p\left(y_{m-1}, y_{m}\right)\right\}-\phi\left(\max \left\{p\left(y_{m-1}, y_{m-1}\right), p\left(y_{m-1}, y_{m}\right)\right\}\right) \\
= & p\left(y_{m-1}, y_{m}\right)-\phi\left(p\left(y_{m-1}, y_{m}\right)\right)\left(b y p_{1}\right)
\end{aligned}
$$

Hence, $p\left(y_{m}, y_{m}\right) \leq p\left(y_{m-1}, y_{m}\right)-\phi\left(p\left(y_{m-1}, y_{m}\right)\right)$.

Also by $P_{1} 0 \leq p\left(y_{m}, y_{m}\right) \leq p\left(y_{m-1}, y_{m}\right)-\phi\left(p\left(y_{m-1}, y_{m}\right)\right)$. Let $m \rightarrow \infty$, using (8) and continuity of $\phi$ we have

$$
\lim _{m \rightarrow \infty} p\left(y_{m}, y_{m}\right)=0
$$

Now, in order to show that $\left\{y_{n}\right\}$ is a Cauchy sequence in the complete metric space, $\left(X, d_{p}\right)$. Assume that $\left\{y_{n}\right\}$ is not Cauchy. Then there exists some $\varepsilon>0$ for which we can find the subsequences $\left\{y_{m(k)}\right\}$ and $\left\{y_{n(k)}\right\}$ of $\left\{y_{n}\right\}$ with $n(k)>m(k)>k$ such that

$$
d_{p}\left(y_{m(k)}, x_{n(k)}\right) \geq \varepsilon
$$

Further, we can choose $n(k)$ corresponding to $m(k)$, in such a way that it is the smallest integer satisfying (10) hence

$$
d_{p}\left(y_{m(k)}, y_{n(k)-1}\right)<\varepsilon
$$

From (10)

$$
\begin{gathered}
\varepsilon \leq d_{p}\left(y_{m(k)}, y_{n(k)}\right) \leq d_{p}\left(y_{m(k)}, y_{n(k)-1}\right)+d_{p}\left(y_{n(k)-1}, y_{n(k)}\right) \\
<\varepsilon+d_{p}\left(y_{n(k)-1}, y_{n(k)}\right) .
\end{gathered}
$$

Hence,

$$
\varepsilon \leq d_{p}\left(y_{m(k)}, y_{n(k)}\right)<\varepsilon+d_{p}\left(y_{n(k)-1}, y_{n(k)}\right)
$$


We know that,

$$
d_{p}\left(y_{n(k)-1}, y_{n(k)}\right)=2 p\left(y_{n(k)-1}, y_{n(k)}\right)-p\left(y_{n(k)}, y_{n(k)}\right)-p\left(y_{n(k)-1}, y_{n(k)-1}\right) .
$$

Let $k \rightarrow \infty$, using (8) and (9), we get

$$
\lim _{k \rightarrow \infty} d_{p}\left(y_{n(k)-1}, y_{n(k)}\right)=0 .
$$

Using (13) in (12), we have

$$
\lim _{k \rightarrow \infty} d_{p}\left(y_{m(k)}, y_{n(k)}\right)=\varepsilon
$$

We, also know that

$$
d_{p}\left(y_{m(k)}, y_{n(k)}\right)=2 p\left(y_{m(k)}, y_{n(k)}\right)-p\left(y_{m(k)}, y_{m(k)}\right)-p\left(y_{n(k)}, y_{n(k)}\right)
$$

Let $k \rightarrow \infty$, using (9) and (14) we get

$$
\lim _{k \rightarrow \infty} d_{p}\left(y_{m(k)}, y_{n(k)}\right)=2 \lim _{k \rightarrow \infty} p\left(y_{m(k)}, y_{n(k)}\right) .
$$

Therefore, we get

$$
\lim _{k \rightarrow \infty} p\left(y_{m(k)}, y_{n(k)}\right)=\frac{\varepsilon}{2}
$$

From the triangular inequality

$$
d_{p}\left(y_{n(k)}, y_{m(k)}\right) \leq d_{p}\left(y_{n(k)}, y_{n(k)+1}\right)+d_{p}\left(y_{n(k)+1}, y_{m(k)+1}\right)+d_{p}\left(y_{m(k)+1}, y_{m(k)}\right)
$$

and

$$
d_{p}\left(y_{n(k)+1}, y_{m(k)+1}\right) \leq d_{p}\left(y_{n(k)+1}, y_{n(k)}\right)+d_{p}\left(y_{n(k)}, y_{m(k)}\right)+d_{p}\left(y_{m(k)}, y_{m(k)+1}\right) .
$$

Let $k \rightarrow \infty$, and using (13) and (14) we get

$$
\lim _{k \rightarrow \infty} d_{p}\left(y_{n(k)}, y_{m(k)}\right) \leq \lim _{k \rightarrow \infty} d_{p}\left(y_{n(k)+1}, y_{m(k)+1}\right)
$$

and

$$
\lim _{k \rightarrow \infty} d_{p}\left(y_{n(k)+1}, y_{m(k)+1}\right) \leq \lim _{k \rightarrow \infty} d_{p}\left(y_{n(k)}, y_{m(k)}\right)
$$

Hence,

$$
\lim _{k \rightarrow \infty} d_{p}\left(y_{n(k)+1}, y_{m(k)+1}\right)=\lim _{k \rightarrow \infty} d_{p}\left(y_{n(k)}, y_{m(k)}\right)=\varepsilon .
$$


By definition of $d_{p}$,

$$
d_{p}\left(y_{m(k)+1}, y_{n(k)+1}\right)=2 p\left(y_{m(k)+1}, y_{n(k)+1}\right)-p\left(y_{m(k)+1}, y_{m(k)+1}\right)-p\left(y_{n(k)+1}, y_{n(k)+1}\right) .
$$

Let $k \rightarrow \infty$, and using (9) we get

$$
\lim _{k \rightarrow \infty} d_{p}\left(y_{m(k)+1}, y_{n(k)+1}\right)=2 \lim _{k \rightarrow \infty} p\left(y_{m(k)+1}, y_{n(k)+1}\right)=\varepsilon .
$$

Which gives

$$
\lim _{k \rightarrow \infty} p\left(y_{m(k)+1}, y_{n(k)+1}\right)=\frac{\varepsilon}{2}
$$

Now, consider

$$
d_{p}\left(y_{m(k)}, y_{n(k)}\right) \leq d_{p}\left(y_{m(k)}, y_{n(k)+1}\right)+d_{p}\left(y_{n(k)+1}, y_{n(k)}\right)
$$

and

$$
d_{p}\left(y_{m(k)}, y_{n(k)+1}\right) \leq d_{p}\left(y_{m(k)}, y_{n(k)}\right)+d_{p}\left(y_{n(k)}, y_{n(k)+1}\right) .
$$

Let $k \rightarrow \infty$, in the above inequalities and using (13) and (14) we get

$$
\varepsilon \leq \lim _{k \rightarrow \infty} d_{p}\left(y_{m(k)}, y_{n(k)+1}\right) \quad \text { and } \quad \lim _{k \rightarrow \infty} d_{p}\left(y_{m(k)}, y_{n(k)+1}\right) \leq \varepsilon .
$$

Therefore,

$$
\lim _{k \rightarrow \infty} d_{p}\left(y_{m(k)}, y_{n(k)+1}\right)=\varepsilon \text {. }
$$

Similarly, we can show that

$$
\lim _{k \rightarrow \infty} d_{p}\left(y_{n(k)}, y_{m(k)+1}\right)=\varepsilon .
$$

Again by using the definition of $d_{p}$, we have

$$
d_{p}\left(y_{m(k)}, y_{n(k)+1}\right)=2 p\left(y_{m(k)}, y_{n(k)+1}\right)-p\left(y_{m(k)}, y_{m(k)}\right)-p\left(y_{n(k)+1}, y_{n(k)+1}\right)
$$

Letting $k \rightarrow \infty$, and using (9) and (18) we get

$$
\lim _{k \rightarrow \infty} d_{p}\left(y_{m(k)}, y_{n(k)+1}\right)=2 \lim _{k \rightarrow \infty} p\left(y_{m(k)}, y_{n(k)+1}\right),
$$

which gives 


$$
\lim _{k \rightarrow \infty} p\left(y_{m(k)}, y_{n(k)+1}\right)=\frac{\varepsilon}{2}
$$

Similarly, we can show that

$$
\lim _{k \rightarrow \infty} p\left(y_{n(k)}, y_{m(k)+1}\right)=\frac{\varepsilon}{2} \text {. }
$$

Now by substituting $x=y_{m(k)}$ and $y=y_{n(k)}$ in (1) we have

$$
\begin{aligned}
p\left(T y_{m(k)}, T y_{n(k)}\right)=p\left(y_{m(k)+1}, y_{n(k)+1}\right) \leq \max \left\{\begin{array}{l}
p\left(y_{m(k)}, y_{n(k)}\right), \frac{1}{2}\left[p\left(y_{m(k)}, y_{m(k)+1}\right)+p\left(y_{n(k)}, y_{n(k)+1}\right)\right), \\
\frac{1}{2}\left[p\left(y_{m(k)}, y_{n(k)+1}\right)+p\left(y_{n(k)}, y_{m(k)+1}\right)\right]
\end{array}\right\} \\
-\phi\left(\max \left\{\begin{array}{l}
p\left(y_{m(k)}, y_{n(k)}\right), \frac{1}{2}\left[p\left(y_{m(k)}, y_{m(k)+1}\right)+p\left(y_{n(k)}, y_{n(k)+1}\right)\right], \\
\frac{1}{2}\left[p\left(y_{m(k)}, y_{n(k)+1}\right)+p\left(y_{n(k)}, y_{m(k)+1}\right)\right]
\end{array}\right\}\right)
\end{aligned}
$$

Letting $k \rightarrow \infty$, and using (8), (15), (17), (20), (21) and using the continuity of $\phi$ we get

$$
\frac{\varepsilon}{2} \leq \max \left\{\frac{\varepsilon}{2}, 0, \frac{\varepsilon}{2}\right\}-\phi\left(\max \left\{\frac{\varepsilon}{2}, 0, \frac{\varepsilon}{2}\right\}\right), \quad \text { hence } \quad \frac{\varepsilon}{2} \leq \frac{\varepsilon}{2}-\phi\left(\frac{\varepsilon}{2}\right)
$$

A contradiction. Thus $\left\{y_{n}\right\}$ is a Cauchy sequence in $\left(X, d_{p}\right)$. Which gives

$$
\lim _{m, n \rightarrow \infty} d_{p}\left(y_{n}, y_{m}\right)=0
$$

Since $\left(X, d_{p}\right)$ is complete so there exists $z \in X$ such that $\lim _{n \rightarrow \infty} d_{p}\left(y_{n}, z\right)=0$, if and only if

$$
p(z, z)=\lim _{n \rightarrow \infty} p\left(y_{n}, z\right)=\lim _{m, n \rightarrow \infty} p\left(y_{n}, y_{m}\right)=\frac{1}{2} \lim _{m, n \rightarrow \infty} d_{p}\left(y_{n}, y_{m}\right)=0 .
$$

(by Lemma 1.3(II) and (22)). This gives,

$$
p(z, z)=\lim _{n \rightarrow \infty} p\left(y_{n}, z\right)=\lim _{m, n \rightarrow \infty} p\left(y_{n}, y_{m}\right)=0 .
$$

Now, applying (1) with $x=y_{n}$ and $y=z$, we have

$$
p\left(T y_{n}, T z\right)=p\left(y_{n+1}, T z\right)
$$




$$
\begin{aligned}
& \leq \max \left\{p\left(y_{n}, z\right), \frac{1}{2}\left[p\left(y_{n}, y_{n+1}\right)+p(z, T z)\right], \frac{1}{2}\left[p\left(y_{n}, T z\right)+p\left(z, y_{n+1}\right)\right]\right\} \\
& -\phi\left(\max \left\{p\left(y_{n}, z\right), \frac{1}{2}\left[p\left(y_{n}, y_{n+1}\right)+p(z, T z)\right], \frac{1}{2}\left[p\left(y_{n}, T z\right)+p\left(z, y_{n+1}\right)\right]\right\}\right)
\end{aligned}
$$

Letting $k \rightarrow \infty$, using (8), (23), Lemma 1.3 (IV) and the continuity of $\phi$ we get

$$
p(z, T z) \leq \frac{1}{2} p(z, T z)-\phi\left(\frac{1}{2} p(z, T z)\right)<\frac{1}{2} p(z, T z)
$$

Which is possible only if $p(z, T z)=0$ and hence $T z=z$.

To show the uniqueness of $z$ consider $z^{*}$ as another fixed point of $T$ then by (1),

$$
p\left(z, z^{*}\right) \leq \max \left\{p\left(z, z^{*}\right), \frac{1}{2}\left[p(z, z)+p\left(z^{*}, z^{*}\right)\right]\right\}-\phi\left(\max \left\{p\left(z, z^{*}\right), \frac{1}{2}\left[p(z, z)+p\left(z^{*}, z^{*}\right)\right]\right\}\right)
$$

By using $P_{1}$ we have $p(z, z) \leq p\left(z, z^{*}\right)$ and $p\left(z^{*}, z^{*}\right) \leq p\left(z, z^{*}\right)$.

Adding above two inequalities, we get

$$
p(z, z)+p\left(z^{*}, z^{*}\right) \leq 2 p\left(z, z^{*}\right) .
$$

Using (25) in (24), we have $p\left(z, z^{*}\right) \leq p\left(z, z^{*}\right)-\phi\left(p\left(z, z^{*}\right)\right)$. Further by using the property of $\phi$ we deduce that $p\left(z, z^{*}\right)=0$ and hence, $z=z^{*}$. Thus $z$ is the unique fixed point of $T$.

Example 2.2 Suppose $X=R^{+}$and $p(x, y)=\max \{x, y\}$; Then $(X, p)$ is a complete partial metric space. Let $T: X \rightarrow X$ be defined by $T x=\frac{x^{2}}{1+x}$ for all $x \in X$ and $\phi:[0, \infty) \rightarrow[0, \infty)$ is such that $\phi(t)=\frac{t}{1+t}$. Assume that $x \geq y$. Then from the contractive condition of Theorem 3.1, we have

$$
\begin{aligned}
p(T x, T y) & \leq \max \left\{p(x, y), \frac{1}{2}\left[p\left(x, \frac{x^{2}}{1+x}\right)+p\left(y, \frac{y^{2}}{1+y}\right)\right], \frac{1}{2}\left[p\left(x, \frac{y^{2}}{1+y}\right)+p\left(y, \frac{x^{2}}{1+x}\right)\right]\right\} \\
& -\phi\left(\max \left\{p(x, y), \frac{1}{2}\left[p\left(x, \frac{x^{2}}{1+x}\right)+p\left(y, \frac{y^{2}}{1+y}\right)\right], \frac{1}{2}\left[p\left(x, \frac{y^{2}}{1+y}\right)+p\left(y, \frac{x^{2}}{1+x}\right)\right]\right\}\right)
\end{aligned}
$$


As $\frac{x^{2}}{1+x}=p\left(\frac{x^{2}}{1+x}, \frac{y^{2}}{1+y}\right) \leq x-\phi(x)=x-\frac{x}{1+x}=\frac{x^{2}}{1+x}$. All the conditions of Theorem 3.1 are true, thus $T$ has a unique fixed point namely, 0 .

In the following theorem we shall obtain a fixed point theorem on partial metric space for a generalized weak contractive type mapping.

Theorem 2.3 Let $(X, p)$ be a complete partial metric space and $T: X \rightarrow X$ be a self-mapping such that for all $x, y \in X$,

$$
\begin{gathered}
\psi(p(T x, T y)) \leq \psi\left(\max \left\{p(x, y), \frac{1}{2}[p(x, T x)+p(y, T y)], \frac{1}{2}[p(x, T y)+p(y, T x)]\right\}\right) \\
-\phi(\max \{p(x, y), p(x, T x)\}),
\end{gathered}
$$

where $\phi:[0, \infty) \rightarrow[0, \infty)$ is a continuous function with $\phi(t)=0$ if and only if $t=0, \psi:[0, \infty) \rightarrow[0, \infty)$ is monotone non-decreasing and continuous function with $\psi(t)=0$ if and only if $t=0$. Then $T$ has a unique fixed point.

Proof: Let $y_{0} \in X$ be fixed. Define a sequence of iterates $\left\{y_{n}\right\}$ in $X$, by $y_{n+1}=T y_{n}$ for all $n \geq 0$. If for some positive integer $m, p\left(y_{m+1}, y_{m}\right)=0$, then by $p_{1}$ and $p_{2}, y_{m}$ is the fixed point of $T$. Hence, assume that $p\left(y_{n+1}, y_{n}\right) \neq 0$ for all $n \geq 0$. Put $x=y_{n}, y=y_{n+1}$ in (26) we have

$$
\begin{aligned}
\psi\left(p\left(y_{n+1}, y_{n+2}\right)\right) \leq \psi\left(\max \left\{\begin{array}{l}
p\left(y_{n}, y_{n+1}\right), \frac{1}{2}\left[p\left(y_{n}, y_{n+1}\right)+p\left(y_{n+1}, y_{n+2}\right)\right], \\
\frac{1}{2}\left[p\left(y_{n}, y_{n+2}\right)+p\left(y_{n+1}, y_{n+1}\right)\right]
\end{array}\right\}\right) \\
-\phi\left(\max \left\{p\left(y_{n}, y_{n+1}\right), p\left(y_{n}, y_{n+1}\right)\right\}\right) .
\end{aligned}
$$

From $p_{4}$, we have $p\left(y_{n}, y_{n+2}\right)+p\left(y_{n+1}, y_{n+1}\right) \leq p\left(y_{n}, y_{n+1}\right)+p\left(y_{n+1}, y_{n+2}\right)$, therefore (27) becomes

$$
\psi\left(p\left(y_{n+1}, y_{n+2}\right)\right) \leq \psi\left(\max \left\{p\left(y_{n}, y_{n+1}\right), \frac{1}{2}\left[p\left(y_{n}, y_{n+1}\right)+p\left(y_{n+1}, y_{n+2}\right)\right]\right\}\right)-\phi\left(p\left(y_{n}, y_{n+1}\right)\right)
$$


If $p\left(y_{n}, y_{n+1}\right) \leq \frac{1}{2}\left[p\left(y_{n}, y_{n+1}\right)+p\left(y_{n+1}, y_{n+2}\right)\right]$. Then (28) becomes

$$
\begin{aligned}
& \psi\left(p\left(y_{n+1}, y_{n+2}\right)\right) \leq \psi\left(\frac{1}{2}\left[p\left(y_{n}, y_{n+1}\right)+p\left(y_{n+1}, y_{n+2}\right)\right]\right)-\phi\left(p\left(y_{n}, y_{n+1}\right)\right) \\
&<\psi\left(\frac{1}{2}\left[p\left(y_{n}, y_{n+1}\right)+p\left(y_{n+1}, y_{n+2}\right)\right]\right) \quad\left(\text { because } \quad \phi\left(p\left(y_{n}, y_{n+1}\right)\right)>0\right)
\end{aligned}
$$

Since $\psi$ is monotone increasing so $p\left(y_{n+1}, y_{n+2}\right) \leq \frac{1}{2}\left[p\left(y_{n}, y_{n+1}\right)+p\left(y_{n+1}, y_{n+2}\right)\right]$, which gives

$$
p\left(y_{n+1}, y_{n+2}\right) \leq p\left(y_{n}, y_{n+1}\right)
$$

If $\frac{1}{2}\left[p\left(y_{n}, y_{n+1}\right)+p\left(y_{n+1}, y_{n+2}\right)\right] \leq p\left(y_{n}, y_{n+1}\right)$

then (28) becomes $\psi\left(p\left(y_{n+1}, y_{n+2}\right)\right) \leq \psi\left(p\left(y_{n}, y_{n+1}\right)\right)-\phi\left(p\left(y_{n}, y_{n+1}\right)\right)$

$$
<\psi\left(p\left(y_{n}, y_{n+1}\right)\right) \quad\left(\therefore \phi\left(p\left(y_{n}, y_{n+1}\right)\right)>0\right)
$$

Hence, we get

$$
p\left(y_{n+1}, y_{n+2}\right) \leq p\left(y_{n}, y_{n+1}\right)
$$

Since $\psi$ is monotone non-decreasing, so in both cases $\left\{p\left(y_{n}, y_{n+1}\right)\right\}$ is monotone decreasing sequence of non-negative real numbers. Hence, there exists a real number $r \geq 0$ such that

$$
\lim _{n \rightarrow \infty}\left\{p\left(y_{n}, y_{n+1}\right)\right\}=r
$$

If $\max \left\{p\left(y_{n}, y_{n+1}\right), \frac{1}{2}\left[p\left(y_{n}, y_{n+1}\right)+p\left(y_{n+1}, y_{n+2}\right)\right]\right\}=p\left(y_{n}, y_{n+1}\right)$.

Then from (28) we have

$$
\psi\left(p\left(y_{n+1}, y_{n+2}\right)\right) \leq \psi\left(p\left(y_{n}, y_{n+1}\right)\right)-\phi\left(p\left(y_{n}, y_{n+1}\right)\right) .
$$

Taking limit as $n \rightarrow \infty$, using (31) and the continuity of $\psi$ and $\phi$ we get,

$$
\psi(r) \leq \psi(r)-\phi(r) \text {, which is a contradiction unless } r=0 \text {. }
$$

If 


$$
\max \left\{p\left(y_{n}, y_{n+1}\right), \frac{1}{2}\left[p\left(y_{n}, y_{n+1}\right)+p\left(y_{n+1}, y_{n+2}\right)\right]\right\}=\frac{1}{2}\left[p\left(y_{n}, y_{n+1}\right)+p\left(y_{n+1}, y_{n+2}\right)\right]
$$

then from (28) we have

$$
\psi\left(p\left(y_{n+1}, y_{n+2}\right)\right) \leq \psi\left(\frac{1}{2}\left[p\left(y_{n}, y_{n+1}\right)+p\left(y_{n+1}, y_{n+2}\right)\right]\right)-\phi\left(p\left(y_{n}, y_{n+1}\right)\right) .
$$

Taking the limit as $n \rightarrow \infty$, using (31) and the continuity of $\psi$ and $\phi$ we get

$\psi(r) \leq \psi(r)-\phi(r)$, which is a contradiction unless $r=0$. Thus $\lim _{n \rightarrow \infty} p\left(y_{n}, y_{n+1}\right)=0$.

Also from $p_{1}$, we get

$$
\lim _{n \rightarrow \infty} p\left(y_{n}, y_{n}\right)=0
$$

Next, we prove that $\left\{y_{n}\right\}$ is a Cauchy sequence in the complete metric space $\left(X, d_{p}\right)$.

Following the steps $(10-21)$ in the proof of Theorem 3.1 and applying (26) with $x=y_{n(k)}$ and $y=y_{m(k)}$ we obtain

$$
\begin{aligned}
\psi\left(p\left(y_{n(k)+1}, y_{m(k)+1}\right)\right) \leq \psi\left(\max \left\{\begin{array}{l}
\left.p\left(y_{n(k)}, y_{m(k)}\right), \frac{1}{2}\left[p\left(y_{n(k)}, y_{n(k)+1}\right)+p\left(y_{m(k)}, y_{m(k)+1}\right)\right]\right\} \\
\frac{1}{2}\left[p\left(y_{n(k)}, y_{m(k)+1}\right)+p\left(y_{m(k)}, y_{n(k)+1}\right)\right]
\end{array}\right\}\right) \\
-\phi\left(\max \left\{p\left(y_{n(k)}, y_{m(k)}\right), p\left(y_{n(k)}, y_{n(k)+1}\right)\right\}\right) .
\end{aligned}
$$

Taking limit as $k \rightarrow \infty$, in (36) we have $\psi\left(\frac{\varepsilon}{2}\right) \leq \psi\left(\frac{\varepsilon}{2}\right)-\phi\left(\frac{\varepsilon}{2}\right)$, which is impossible since $\phi\left(\frac{\varepsilon}{2}\right)>0$. Hence, $\left\{y_{n}\right\}$ is a Cauchy sequence in the complete metric space $\left(X, d_{p}\right)$. So there exists some $z \in X$ such that $\lim _{n \rightarrow \infty} d_{p}\left(y_{n}, z\right)=0$ if and only if

$$
p(z, z)=\lim _{n \rightarrow \infty} p\left(y_{n}, z\right)=\lim _{m, n \rightarrow \infty} p\left(y_{n}, y_{m}\right)=\frac{1}{2} \lim _{m, n \rightarrow \infty} d_{p}\left(y_{n}, y_{m}\right)=0
$$

Hence

$$
p(z, z)=\lim _{n \rightarrow \infty} p\left(y_{n}, z\right)=\lim _{m, n \rightarrow \infty} p\left(y_{n}, y_{m}\right)=0 .
$$


To show that $z$ is the fixed point of $T$ put $x=y_{n}$ and $y=z$ in (26).

$$
\begin{aligned}
\psi\left(p\left(y_{n+1}, T z\right)\right) \leq \psi\left(\operatorname { m a x } \left\{p\left(y_{n}, z\right)\right.\right. & \left.\left., \frac{1}{2}\left[p\left(y_{n}, y_{n+1}\right)+p(z, T z)\right], \frac{1}{2}\left[p\left(y_{n}, T z\right)+p\left(z, y_{n+1}\right)\right]\right\}\right) \\
& -\phi\left(\max \left\{p\left(y_{n}, z\right), p\left(y_{n}, y_{n+1}\right)\right\}\right) .
\end{aligned}
$$

Letting $n \rightarrow \infty$, in (38) we have $\psi(p(z, T z)) \leq \psi\left(\frac{1}{2} p(z, T z)\right)$. Which forces $p(z, T z)=0$ and hence $T z=z$. Thus $z$ is the fixed point of $T$. In order to prove the uniqueness of $z$ consider $z^{*}$ as another fixed point of $T$ then for taking $x=z$ and $y=z^{*}$ in (26) we have

$$
\begin{gathered}
\psi\left(p\left(T z, T z^{*}\right)\right)=\psi\left(p\left(z, z^{*}\right)\right) \leq \psi\left(\max \left\{p\left(z, z^{*}\right), \frac{1}{2}\left[p(z, z)+p\left(z^{*}, z^{*}\right)\right], \frac{1}{2}\left[p\left(z, z^{*}\right)+p\left(z^{*}, z\right)\right]\right\}\right) \\
-\phi\left(\max \left\{p\left(z, z^{*}\right), p(z, z)\right\}\right) .
\end{gathered}
$$

Thus $\psi\left(p\left(z, z^{*}\right)\right) \leq \psi\left(p\left(z, z^{*}\right)\right)-\phi\left(p\left(z, z^{*}\right)\right)$. Which is possible only if $p\left(z, z^{*}\right)=0$. By using $p_{1}$ and $p_{2}$ we get $z=z^{*}$. Thus $T$ has a unique fixed point.

Now, we consider an example to support the usability of Theorem 2.3.

Example 2.4 In Example 2.2 if we define $\psi:[0, \infty) \rightarrow[0, \infty)$ by $\psi(t)=t$, for all $t \in[0, \infty)$. Then the contractive condition of Theorem 2.3 is satisfied and we have 0 as the unique fixed point of $T$.

Corollary 2.5 Let $X$ be a complete partial metric space. Let $T: X \rightarrow X$ be a self-mapping such that all elements $x, y$ of $X$, satisfy

$$
\begin{gathered}
\psi\left(p\left(T^{m} x, T^{m} y\right)\right) \leq \psi\left(\max \left\{\begin{array}{l}
p(x, y), \frac{1}{2}\left[p\left(x, T^{m} x\right)+p\left(y, T^{m} y\right)\right], \\
\frac{1}{2}\left[p\left(x, T^{m} y\right)+p\left(y, T^{m} x\right)\right]
\end{array}\right\}\right) \\
-\phi\left(\max \left\{p(x, y), p\left(x, T^{m} x\right)\right\}\right),
\end{gathered}
$$

where $m$ is a positive integer and $\phi, \psi$ are as defined in Theorem 2.3. Then $T$ has a unique fixed point in $X$.

Proof: Put $S=T^{m}$ in (39) we have, 


$$
\begin{aligned}
\psi(p(S x, S y)) \leq & \psi\left(\max \left\{p(x, y), \frac{1}{2}[p(x, S x)+p(y, S y)], \frac{1}{2}[p(x, S y)+p(y, S x)]\right\}\right) \\
& -\phi(\max \{p(x, y), p(x, S x)\})
\end{aligned}
$$

Hence by Theorem 2.3, $S$ has a unique fixed point $z$ that is, $S z=z$ since $S=T^{m}$ so $T^{m} z=z$ which gives $T\left(T^{m} z\right)=T^{m+1} z=T^{m}(T z)=S(T z)=T z$, which shows that $T z$ is also a fixed point of $S$. Since $S$ has a unique fixed point so $T z=z$. Hence, $z$ is the fixed point of $T$. Condition (39) implies the uniqueness of $z$.

Conclusion: We have generalized the theorem proved by Rhoades[13] for a self map on a complete partial metric space and we obtain Matthews' generalization of Banach's contraction principle as a special case of the Theorem 3.1. Moreover, a fixed point theorem for a self map defined for partial metric space satisfying a generalized $(\psi, \phi)$-weak contractive conditions is also proved.

\section{References}

[1] S. G. Matthews, Partial metric spaces. Research Report 212. Department of Computer Science, University of Warwick, 1992.

[2] S. G. Matthews, Partial metric topology, General topology and its appl. Ann New York Acad of Sci.728 (1994) 183-197.

[3] E. Karapinar, Generalizations of Caristi Kirk's theorems on partial metric spaces, Fixed Point Theory and Application (2011); doi:10.1186/1687-1812-2011-4.

[4] I. Altun and A. Erduran, Fixed point theorem for monotone mappings on partial metric spaces, Fixed point theory appl. Vol.2011, Dec 24 (2010) Article ID 508730, 10 pages.

[5] T. Abdeljawed, Fixed points for generalized weakly contractive mappings in partial metric spaces, Mathematical and Computer modelling. 54 (2011) 2923-2927.

[6] E. Karapinar and U. Yuskel, Some common fixed point theorems in partial metric spaces. J. of Appl. Mathematics. Vol 2011 (2011), Article ID 263621,16 pages.

[7] T. Abdeljawed, E. Karapiner and K. Tas, A generalized contraction principle with control function on partial metric spaces, Computers and Mathematics with Appl. 63 (2012) 716-719.

[8] T. Abdeljawed, E. Karapiner and K. Tas, Existence and uniqueness of a common fixed point on partial metric spaces, Appl Math Lett. 24 (2011) 1900-1904.

[9] B. S. Choudhury and A. Kundu, Weak contractions in partial metric spaces, Department of Mathematics Bengal engineering and Sci Uni Shibpur, P.O-B garden Howrah-711103. India.

[10] D. W. Boyd and T. S. W. Wong, On nonlinear contractions, Proc Amer Math Soc. 69; 20; 458-464.

[11] Y. I. Alber and S. Guerre-Delabriere, Principles of weakly contractive maps in Hilbert spaces, in: New results in operator theory,in: I.GoldbergYu.Lyubich(Eds), Advances and Appl. 98, Birkhäuser, Basel, (1997) 7-22.

[12] Q. Zhang and Y. Song, Fixed point theory for generalized-weak contractions. Appl Math Lett. 2008, 22 (2009) 75-78.

[13] B. E. Rhoades, Some theorems on weakly contractive maps. Nonlinear Anal. 47 (2001) 2683-2693.

[14] M. Jain, N. Gupta and S. Kumar, Coupled fixed point results for mappings involving (lalpha, Ipsi)-weak contractions in ordered metric spaces and applications, The Journal of Mathematics and Computer Science, 10 (2012), 23-46. 